\title{
Mucoadhesive Film Embedded with Acyclovir Loaded Biopolymeric Nanoparticles: In vitro Studies
}

\author{
Anroop Balachandran Nair ${ }^{1 *}$, Abbas Ahmed Al-ghannam¹, Bandar Essa Al-Dhubiab , Azza Ali Hasan² \\ 'Department of Pharmaceutical Sciences, College of Clinical Pharmacy, King Faisal University, Al-Ahsa, SAUDI ARABIA. \\ 2Department of Pharmaceutics and Industrial Pharmacy, Faculty of Pharmacy, Zagazig University, Zagazig, EGYPT
}

\begin{abstract}
Objective: The efficiency of acyclovir, a potent antiviral agent, is mainly limited by its low permeability. The purpose of this investigation was to formulate and assess nanoparticle impregnated buccal films to improve acyclovir permeability and thereby enhance bioavailability. Methods: Acyclovir was incorporated into the polymeric materials and formulated as nanoparticles. The prepared nanoparticles were then loaded into various films (F5-F7) prepared with varying quantities of hydroxyethyl cellulose and Eudragit $R L$ 100. The prepared films were evaluated for physico-mechanical characters, mucoadhesion, swelling, in vitro acyclovir release and ex vivo diffusion. Results: The films so prepared showed adequate mucoadhesive strength and excellent physico-mechanical properties. Rapid hydration of films (>35\%) were observed in $15 \mathrm{~min}$, followed by a relatively slower phase of hydration which continued till $60 \mathrm{~min}$. In vitro release profile exhibited biphasic pattern, while the rate of acyclovir release was higher with film F7. Prepared films exhibited either first order (F5, control) or Higuchi model (F6, F7) kinetics. The enhancement in acyclovir permeation in F5, F6 and F7 loaded films were 2.2, 4.1 and 6.7 folds, respectively, when compared
\end{abstract}

to control. Conclusion: This study concludes that the drug loaded nanoparticles impregnated buccal film could be an alternative approach to enhance the oral bioavailability of acyclovir, and need to be proved in vivo.

Key words: Acyclovir, buccal, film, Nanoparticles, permeation, release studies.

Correspondence:

Anroop B Nair*

Department of Pharmaceutical Sciences, College of Clinical Pharmacy, King Faisal University, Al-Ahsa, SAUDI ARABIA.

Tel: 966-536219868

Fax: 966-3-5817174

E-mail: anair@kfu.edu.sa

DOI: 10.5530/jyp.2017.9.19

\section{INTRODUCTION}

Buccal route of drug delivery offers most promising alternative to oral route because of its potential to overcome first-pass hepatic metabolism as well as gastrointestinal-tract limitations. ${ }^{1}$ In addition, the buccal mucosa is comparatively permeable and is readily accessible for self-medication. ${ }^{2}$ The potential of this route to augment trans-mucosal transport of therapeutic agents and delivering it into blood has been demonstrated for several poorly oral bioavailable drugs. ${ }^{3,4}$ Many types of mucoadhesive dosage forms have been designed and investigated for buccal delivery in the past decade. However, efficiency of these formulations primarily depend on their adhesion with mucosa to enable effective drug release and transport across the membrane. The concept of mucoadhesive polymeric buccal films has garnered considerable importance among drug delivery scientists owing to their advantages like ease of adherence to mucosa, high flexibility, higher patient compliance, controlled release of drug, prolonged residence time etc. ${ }^{5,6}$ In this context, polymeric nanoparticles impregnated buccal films have been extensively studied and proved to be an effective approach to enhance bioavailability of drug molecules in addition to its potential to provide sustained release over an extended period of time. ${ }^{7,8}$ Recently, the potential of polymeric nanospheres of selegiline impregnated buccal films in enhancing the oral bioavailability has been demonstrated. ${ }^{9}$

Acyclovir is one of the potent antiviral drugs used for management of herpes simplex viral infections, Varicella zoster as well as Herpes zoster. ${ }^{10}$ Different dosage forms such as tablets (oral), creams (topical) and suspensions (parenteral) of acyclovir are available with limited efficiency. The systemic availability of acyclovir following oral and topical therapy is very low than the therapeutic requirement. The treatment of herpes infections with acyclovir involves repeated oral administration of acyclovir due to very poor oral bioavailability (15\%) and small elimination half-life $(3 \mathrm{~h}) .{ }^{10}$ The delivery of this active is primarily limited by its biopharmaceutical properties and physiological nature of the membrane. Few approaches have been attempted in the last decade to overcome issues with this drug, but resulted with limited success. ${ }^{11-13}$ In this context, the buccal route could be explored as an alternate to oral therapy of acyclovir by enhancing the absorption, bioavailability and provide effective systemic delivery. It is hypothesized that embedding polymeric nanoparticles of acyclovir in buccal film could enhance the efficiency of this drug molecule. The present study assessed the potential of developing mucoadhesive buccal films embedded with biodegradable polymeric nanoparticles of acyclovir with the objective of improving bioavailability. The mucoadhesive buccal films of acyclovir was fabricated using varying concentrations of hydroxyethyl cellulose (HEC) as mucoadhesive agent, Eudragit RL 100 as a film former, propylene glycol as plasticizer and polyvinyl alcohol as backing membrane.

\section{MATERIAL AND METHODS}

\section{Materials}

Acyclovir, hydroxyethyl cellulose (HEC), poly (D,L-lactide-co-glycolide) (PLGA, 50:50, Mw 7000-17000 dalton), polyvinyl alcohol (PVA; Mowiol ${ }^{\circ}$ 40-88, Mw 205,000), polyvinylpyrrolidone (K 30, Mw 40,000), propylene glycol (PG), dichloromethane, acetonitrile, ethanol and acetic acid 
were purchased from Sigma Aldrich St. Louis, MO, USA. Eudragit RL 100 was obtained from Evonik, Darmstadt, Germany.

\section{Analysis}

Amount of acyclovir in samples were quantified by Ultraviolet (UV) spectroscopy (Shimadzu, Japan). Calibration curve of acyclovir was prepared by dissolving accurately weighed amount of drug and dissolving in phosphate buffered saline (PBS, pH 7.4) in volumetric flask. This stock solution (PBS) was further diluted to various concentrations ranging from $1-500 \mu \mathrm{g} / \mathrm{ml}$. For sample analysis, absorbance was measured at 250 $\mathrm{nm}\left(\lambda_{\max }\right)$ by UV Spectrophotometer, using PBS as blank. The method is validated to avoid any interefence of extracts in permeation studies.

\section{Preparation of nanoparticles}

Nanoparticles were prepared by solvent evaporation technique using chloroform-dichloromethane. ${ }^{14}$ Briefly, chloroform: dichloromethane (20:30 V/V) were measured accurately and mixed well. ${ }^{15}$ Acyclovir and PLGA (1:20 W/W) were weighed and dissolved in the solvent mixture $(10 \mathrm{~mL})$ and added to known volume of PVA solution $(2 \% \mathrm{~W} / \mathrm{V}, 20$ $\mathrm{mL}$ ) and homogenized for $2 \mathrm{~min}$ at 13,000 rpm. The system was further stirred for $2 \mathrm{~h}(600 \mathrm{rpm})$ and allowed to stabilize for $1 \mathrm{~h}$. Then the supernatant liquid was decanted and centrifuged $\left(2 \mathrm{~min}\right.$ at $13416 \times \mathrm{g}$ at $\left.25^{\circ} \mathrm{C}\right)$ to obtain nanoparticles. The product was washed few times and freeze dried for $24 \mathrm{~h}$.

\section{Evaluation of nanoparticles}

The percentage yield of nanoparticles was determined using the formula: ${ }^{16}$

$$
\% \text { Yield }=\frac{\text { Practical Yield }}{\text { Theoretical Yield }} \square 100
$$

Entrapment efficiency was determined by weighing required amount of nanoparticles $(50 \mathrm{mg}$ ), added to PBS and sonicated for $24 \mathrm{~h}$. Then the solution was centrifuged $(13416 \times \mathrm{g})$, supernatant was used for UV analysis. The amount of drug loaded was determined as; Amount of drug/ Amount of nanoparticles $\times 100$. The percentage entrapment efficiency (\%EE) was computed by the equation: ${ }^{16}$

$$
\% \mathrm{EE}=\frac{\mathrm{C}_{\mathrm{t}} \square \mathrm{C}_{\mathrm{r}}}{\mathrm{C}_{\mathrm{t}}} \square 100
$$

where $\mathrm{C}_{\mathrm{t}}$ is total and $\mathrm{C}_{\mathrm{r}}$ is free acyclovir concentration.

Particle size and distribution of nanoparticles were measured using Zetasizer (Malvern, USA).

\section{Preparation of mucoadhesive films}

The required amount of Eudragit RL 100 was dissolved in ethyl alcohol. Amount of Eudragit RL 100 added for film forming was gradually increased (as shown in Table 1) to optimize the Eudragit content required to form a film with desired physicomechanical properties. HEC was dispersed separately in water containing PG and added to Eudragit solution under magnetic stirring. Drug loaded nanoparticles was added to the above mixture and stirred to ensure uniform distribution of nanoparticles. The dispersion so formed was sonicated for $15 \mathrm{~min}$ and casted on to a petridish and incubated at $37^{\circ} \mathrm{C}$ for drying $(24 \mathrm{~h})$.

\section{Preparation of backing membrane}

PVA aqueous solution $(4 \% \mathrm{~W} / \mathrm{V})$ was prepared and casted on a thin sheet and allowed to dry for $12 \mathrm{~h}$ at $40{ }^{\circ} \mathrm{C}$ in an oven. Drug loaded buccal films were casted over the backing membranes by solvent casting. ${ }^{17}$

\section{Evaluation of films}

Quantification of drug in the prepared films were carried out by cutting a film of size $1 \times 1 \mathrm{~cm}^{2}$ and kept in glass vial contain PBS. The solution was mixed for $5 \mathrm{~h}$ by continuous shaking on a water bath, filtered and amount was measured using UV spectrometer. Thickness of the film was noted making use of a screw gauge. The thickness was measured at six different locations. ${ }^{18}$ Folding endurance was measured by folding films $\left(4 \mathrm{x} 4 \mathrm{~cm}^{2}\right)$ at same location repeatedly making an angle of $180^{\circ}$ till it breaks. For $\mathrm{pH}$ determination, film of size of $1 \times 1 \mathrm{~cm}^{2}$ was taken and dipped in water $(5 \mathrm{~mL})$ for 30 minutes. Then the film was removed, surface liquid was cleaned and $\mathrm{pH}$ was measured using a flat surface electrode. ${ }^{18}$

Mucoadhesive studies were carried out using a standard texture analyzer. ${ }^{18}$ Male white rabbits $(2.5-3.0 \mathrm{~kg}$ ) cheek pouch was used to measure the mucoadhesive strength (IAEC/SSP/16/PR-013). Rabbit buccal mucosa was surgically removed from the oral cavity using scalpel and scissors to separate the connective tissue. The AC electrical resistance of the buccal mucosa was measured using a wave form generator and a digital multimeter (Agilent Technologies, Santa Clara, CA, USA). The prepared films were cut into small pieces and fixed to probe of a texture analyzer using cyanoacrylate adhesive. The rabbit epithelium mucosa was mounted on the stationary platform. The assembly containing the rabbit cheek mucosa was filled with $2 \mathrm{~mL}$ of the buffer solution to keep the mucosa wet during the contact period. The movable probe of the texture analyzer was lowered until it made contact with the mucosa. The contact time between the cheek mucosa and film was $1 \mathrm{~min}$. Measurements were obtained using the following parameters: pre-test speed: $0.5 \mathrm{~mm} / \mathrm{s}$; test / post-test speed: $0.5 \mathrm{~mm} / \mathrm{s}$; applied force $1 \mathrm{~N}$. The force needed to separate film from the membrane was measured. The mucoadhesive strength was taken as the maximum amount of force required to detach the film from the rabbit mucosa.

Percentage hydration of prepared films were measured by cutting films $\left(1 \times 1 \mathrm{~cm}^{2}\right)$, weighed $\left(\mathrm{w}_{1}\right)$ and kept on a stainless steel mesh. This was then immersed in PBS for specific periods (15, 30, 45, $60 \mathrm{~min})$. At these time intervals the wire mesh was removed from the buffer, extra water was wiped and determined the weight $\left(\mathrm{w}_{2}\right) .{ }^{4}$ Percent hydration was calculated as;

In vitro release

$$
\text { Percentage hydration }=\frac{\mathrm{W}_{2} \square \mathrm{W}_{1}}{\mathrm{~W}_{2}} \square 100
$$

The film size $\left(2 \times 1 \mathrm{~cm}^{2}\right)$ was cut and pasted on a glass plate and placed inside a beaker $(100 \mathrm{~mL})$ contain PBS $(50 \mathrm{~mL})$. The medium was retained at $37 \pm 1^{\circ} \mathrm{C}$ and stirred at speed of $50 \mathrm{rpm}$. Samples were withdrawn at scheduled time periods, exchanged with new medium, filtered and analyzed. ${ }^{19}$ The acyclovir release data was then evaluated using mathematical models like zero-order, first-order, Hixson-Crowell, Higuchi, and Korsmeyer-Peppas models.

\section{Ex-vivo permeation studies}

The rate and extend of mucosal permeation of acyclovir and/or nanoparticles across the rabbit buccal mucosa (permeation barrier) was determined on a Franz diffusion cell. ${ }^{20}$ The excised rabbit buccal mucosa membrane of specific integrity was fixed on the diffusion cell such that the smooth surface facing donor. The prepared films were cut into small sections $\left(0.6 \mathrm{~cm}^{2}\right)$ and positioned on the surface of buccal epithelium. Receptor compartment with a capacity of $5 \mathrm{~mL}$ was filled with PBS. The temperature was maintained at $37 \pm 0.2^{\circ} \mathrm{C}$, while the media was stirred at $50 \mathrm{rpm}$. Samples were drawn $(1 \mathrm{~mL})$ at specific time periods, sonicated for $24 \mathrm{~h}$ and centrifuged $(13416 \times \mathrm{g})$ for $10 \mathrm{~min}$ to avoid the interference of some biological extracts from excised buccal tissues in UV absorbance during analysis. The samples were further diluted and analyzed using UV spectrophotometer. 
Table 1: Composition of the prepared mucoadhesive buccal films

\begin{tabular}{ccccc}
\hline Formulation code & Acyclovir $(\mathrm{mg})$ & HEC $(\mathrm{mg})$ & Eudragit RL 100 $(\mathrm{mg})$ & PG $(\mathrm{mg})$ \\
\hline F1 & - & 750 & 500 & 100 \\
F2 & - & 500 & 1000 & 100 \\
F3 & - & 250 & 1500 & 100 \\
F4 & - & 100 & 2000 & 100 \\
F5 & $14^{\star}$ & 100 & 2000 & 100 \\
F6 & $28^{*}$ & 100 & 2000 & 100 \\
F7 & $42^{*}$ & 100 & 2000 & 100 \\
Control & $28^{* *}$ & 100 & 2000 & 100 \\
\hline
\end{tabular}

${ }^{\star}$ Incorporated nanoparticles equivalent to acyclovir. ${ }^{*}$ Incorporated free acyclovir.

Table 2: Physico-mechanical properties of films (mean \pm SD)

\begin{tabular}{cccccc}
\hline $\begin{array}{c}\text { Formulation } \\
\text { code }\end{array}$ & $\begin{array}{c}\text { Thickness } \\
(\mathbf{n m})\end{array}$ & $\mathrm{pH}$ & $\begin{array}{c}\text { Folding } \\
\text { Endurance }\end{array}$ & \% drug content & $\begin{array}{c}\text { Mucoadhesive } \\
\text { strength (N) }\end{array}$ \\
\hline F4 & $203 \pm 22$ & $6.2 \pm 0.35$ & $287 \pm 16$ & - & $6.8 \pm 0.35$ \\
F5 & $251 \pm 39$ & $6.4 \pm 0.26$ & $306 \pm 19$ & $92.37 \pm 3.52$ & $6.5 \pm 0.42$ \\
F6 & $283 \pm 32$ & $6.5 \pm 0.30$ & $311 \pm 24$ & $93.91 \pm 1.65$ & $5.9 \pm 0.46$ \\
F7 & $311 \pm 40$ & $6.3 \pm 0.22$ & $333 \pm 35$ & $95.15 \pm 2.26$ & $6.3 \pm 0.51$ \\
Control & $264 \pm 26$ & $6.8 \pm 0.41$ & $277 \pm 15$ & $94.62 \pm 3.91$ & $6.7 \pm 0.58$ \\
\hline
\end{tabular}

Table 3: Regression coefficients assessed by different kinetic models for acyclovir release from films

\begin{tabular}{cccccc}
\hline Formulation code & Zero order & First Order & Higuchi & Hixson-Crowell & Korsmeyer-Peppas \\
\hline F5 & 0.9235 & 0.9922 & 0.9804 & 0.8043 & 0.9715 \\
F6 & 0.9379 & 0.9641 & 0.9869 & 0.8251 & 0.9787 \\
F7 & 0.8990 & 0.9763 & 0.9935 & 0.8103 & 0.9768 \\
Control & 0.8724 & 0.9909 & 0.9423 & 0.7984 & 0.9451 \\
\hline
\end{tabular}

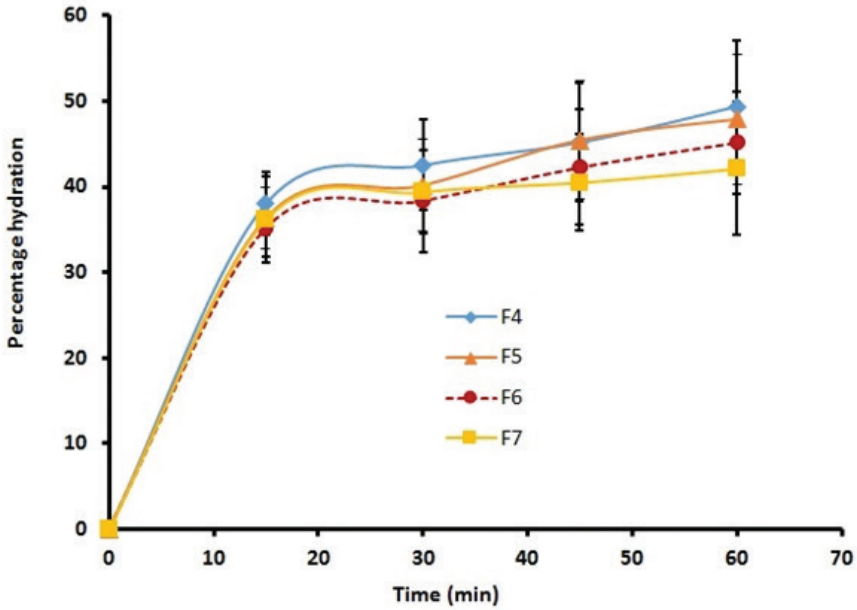

Figure. 1: The percentage hydration pattern of the prepared buccal films for a period of $1 \mathrm{~h}$ determined using $1 \mathrm{~cm} \times 1 \mathrm{~cm}$ of the film. The value represents average of six trials \pm SD.

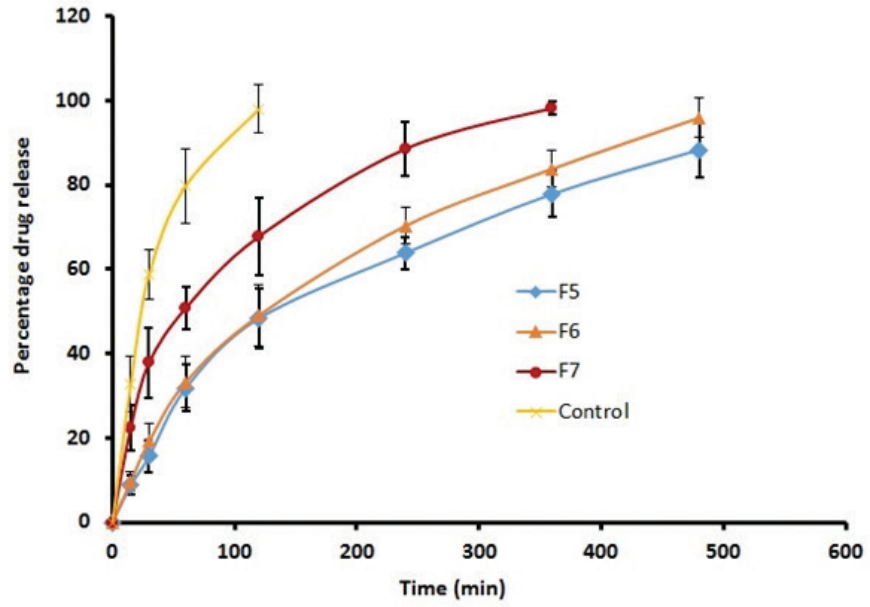

Figure. 2: Comparison of the cumulative percentage of acyclovir released from different buccal films at various time intervals. The value represents average of six trials \pm SD. 


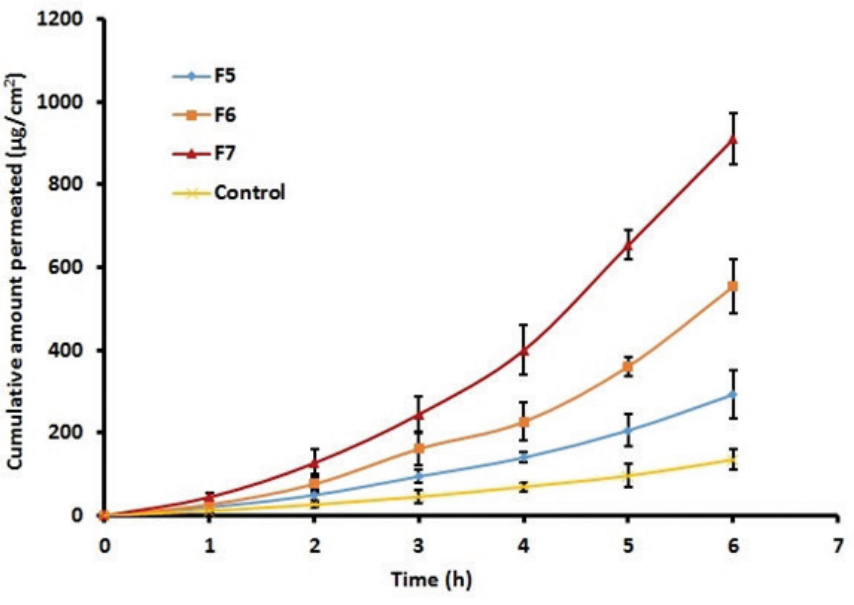

Figure. 3: Comparison of ex vivo permeation of acyclovir from different buccal films at various time intervals. The value represents average of six trials \pm SD.

\section{Data analysis}

A plot of total amount of acyclovir transported through the membrane versus time was made and slope was projected as flux. Unpaired t-test was carried out for conducting statistical analysis using GraphPad software. $P$ value of less than 0.05 was considered as the level of significance. ${ }^{21}$ Mean value and standard errors were calculated using values of six trials.

\section{RESULTS AND DISCUSSION}

Acyclovir is an amphoteric low molecular weight (MW=225 Da) drug having both weak acidic and basic groups with reported partition coefficient $(\log \mathrm{P}=-1.56)$, aqueous solubility $(1.5 \mathrm{mg} / \mathrm{mL})$ and $\mathrm{pKa}$ values (2.16 and 9.04). ${ }^{22}$ In this context, it is presumed that permeation of acyclovir through the biological membrane could be relatively low. Hence, the drug was first enclosed in a biodegradable polymer (PLGA) by formulating into nanoparticles. It is known that the percentage entrapment efficiency gives the indication of entrapped drug in nanoparticles. The highest yield and entrapment was observed when a weight ratio of 1:20 (drug: polymer) was used. In this case, the percentage yield, entrapment efficiency and drug loading were $82.31 \pm 5.91 \%, 64.81 \pm 4.72 \%$ and 4.06 $\pm 1.12 \%$, respectively, and this formulation was used for further studies. In addition, the particle size of the prepared nanoparticles were in the range of 150-400 $\mathrm{nm}$ with a typical size of $220 \pm 40 \mathrm{~nm}$.

In the next stage, buccal films were prepared using HEC as mucoadhesive agent and Eudragit as film former. The selection of these polymers in the current investigation is based on earlier studies. ${ }^{16,17}$ Moreover, HEC is a common excipient used in various pharmaceutical formulations. ${ }^{21}$ The composition of the prepared films is summarized in Table 1. The concentration of HEC (1-7.5\% W/V) and Eudragit (5-20\% W/V) were varied to obtain an appropriate buccal film for clinical use. In case of formulation F1, the film formed was defective as it could not be peeled, while F2 and F3 do not provide adequate strength and was sticky as well. However, composition of F4 was ideal and form a good film. In this formulation the concentration of HEC was $1 \%(\mathrm{~W} / \mathrm{V})$ while the Eudragit content was $20 \%(\mathrm{~W} / \mathrm{V})$. This composition was further incorporated with three different amounts of acyclovir loaded nanoparticles $(0.5 \mathrm{mg} /$ $\mathrm{cm}^{2}$ area, $1 \mathrm{mg} / \mathrm{cm}^{2}$ area and $1.5 \mathrm{mg} / \mathrm{cm}^{2}$ area in $\mathrm{F} 5, \mathrm{~F} 6$ and $\mathrm{F} 7$, respectively) as described in Table 1. Control films were prepared using same polymers in a similar experimental conditions, while the nanoparticles were exchanged with free drug. The concentration of propylene glycol was optimized by varying concentrations between $0.5-2 \% \mathrm{w} / \mathrm{v}$. Thus formulated films were further fixed to the backing membrane prepared using aqueous solution of PVA $(4 \% \mathrm{~W} / \mathrm{V})$.

The films prepared were then evaluated for various physico-mechanical properties. It was found that the prepared films (F4-F7 and control) were transparent, soft, homogenous and peelable. Determination of film thickness is also important to assure good adhesion of film with mucous membrane as well as the uniformity of drug in the film. The prepared films thickness were in the range of 180 to $350 \mathrm{~nm}$ (Table 2). These results showed that formulation F4 (without nanoparticles) had a thinnest film and formulation F7 had thick film. Among nanoparticles loaded films (F5, F6 and F7), the thickness increases with increase in drug content of acyclovir loaded nanoparticles. Certainly, the measured thickness of prepared films (F4-F7 and control) is ideal for application on the buccal mucosa and for bioadhesion. ${ }^{18} \mathrm{pH}$ of the buccal film was measured to ensure optimal $\mathrm{pH}$ for oral application and avoid any damage to the buccal mucosa. Indeed, prepared films (F4-F7 and control) showed a $\mathrm{pH}$ near to 6 .

Mechanical strength and flexibility of prepared films were evaluated by measuring the folding endurance. The data observed in Table 2 suggest that the prepared buccal films (F4-F7 and control) possess adequate folding endurance and can provide easy application on the site of administration. Further, formulations were evaluated for drug content i.e., the amount of drug loaded in the buccal films. Uniform dispersion of nanoparticles in film means the drug is homogeneously distributed in the film. It can be seen from Table 2 that the drug content do not vary among the formulations and is in the range of limits $(100 \pm 10 \% \mathrm{w} / \mathrm{w})$. This comparable values in drug content suggest that the variation in acyclovir loaded nanoparticles have little or no effect on drug content, in the present experimental settings. The low standard deviation also signify that the drug was uniformly dispersed within the film.

The measurement of mucoadhesive strength is likely to provide real time effect of binding of films with buccal mucosa. This is important as the film has to adhere and retain on buccal mucosa for effective delivery of actives. The mucoadhesive strength measured with various formulations were summarized in Table 2 . No significant difference $(P>0.05)$ between mucoadhesive strength (5.9 -6.8 N) of films (F4, F5, F6, F7 and control) also suggest that the incorporation of free drug or nanoparticles have little or no effect. Based on the evidences from literature, it is likely that the values exhibited by the prepared films possess adequate buccoadhesive strength to withstand pressure due to movements in the buccal cavity.

Hydration of films is a prerequisite for adhesion and release of drug molecules. ${ }^{23}$ Following application, the films absorb water and hydrate itself to provide good adhesion in addition helps in separation and diffusion of particles (nanoparticles in this case). However, hydration is highly influenced by polymer, crosslinking as well as interaction between nanoparticles and polymers. ${ }^{24,25}$ The swelling index of all the prepared films (F4, F5, F6 and F7) were comparable (Figure 1). As indicated from Figure 1 , all the prepared films showed rapid hydration in the initial $15 \mathrm{~min}$ of the study, which is in agreement with several earlier studies., 26 This was followed by a relatively slower phase of hydration which continued till $60 \mathrm{~min}$. The prepared films comprised of a combination of hydrophilic (HEC) and hydrophobic (Eudragit) polymer and the hydrophilic polymer is responsible for hydration. However, the greater amount of Eudragit (in the composition) prevent higher hydration, which in turn maintain the integrity and mechanical stability of films.

The prepared films were impregnated with nanoparticles, and as a prerequisite, these nanoparticles must be released from the film and transport through the membrane. The release of nanoparticles from formulations is governed by the separation of nanoparticles from the polymer matrix and their diffusion from the matrix. ${ }^{27}$ Percentage of drug released from different films [F5, F6, F7 and control (drug incorporated films)] 
are depicted in Figure 2. It is evident from Figure 2 that all formulations exhibited a biphasic release pattern. The drug release was rapid in the initial $2 \mathrm{~h}$ and were $48.37 \pm 7.12 \%, 49.05 \pm 7.24 \%, 67.79 \pm 9.16 \%$ and $97.98 \pm 5.63 \%$ in F5, F6, F7 and control (drug incorporated films), respectively. The high release observed could be attributed to the presence of hydrophilic polymer (HEC) in the films, which would have allowed the diffusion of water more rapidly and helped in release of nanoparticles. The rapid release was followed by slower release in nanoparticles loaded films, while in control drug release was complete in $2 \mathrm{~h}$. Among nanoparticles loaded films, the percentage release was relatively high with film F7 (with highest drug content). However, the percentage drug release was comparable $(P>0.05)$ in film F5 and F6.

The in vitro release data were applied to various kinetic models such as zero-order, first-order, Hixson-Crowell, Higuchi, and Korsmeyer-Peppas models to predict the drug release mechanism and kinetics. It is evident from Table 3 that the calculated regression $\left(r^{2}\right)$ values were different for prepared films. Briefly, when log percent of drug remaining to be release versus time was plotted in accordance with first order equation, a straight line was obtained $\left(\mathrm{r}^{2}>0.99\right)$ for formulation F5 and control. These values indicated that the drug release followed first order kinetics, where the drug release was dependent upon acyclovir concentration in the polymer matrix. However, in case of formulations F6 and F7, the drug release mechanism was diffusion controlled as plots of the amount releases versus square root of time was found to be linear. Overall, the kinetics data suggest that the release was first order when the acyclovir concentration was low $\left(0.5 \mathrm{mg} / \mathrm{cm}^{2}\right)$ while it followed Higuchi model at high drug concentration $\left(1 \mathrm{mg} / \mathrm{cm}^{2}\right)$.

Permeation study is done usually to confer the transport of drug across permeation barriers. The permeation of drug molecules into and through a biological barrier is influenced by thickness of the membrane and its composition, and the specific passage accessible for drug permeation. ${ }^{28}$ The amount of acyclovir incorporated in the films was determined based on its standard human dose. ${ }^{29}$ The permeation profiles of drug loaded films (F5, F6, F7 and control) are shown in Figure 3. It is evident from Figure 3 that the drug permeation increased with duration of time. Similarly, the increase in drug content in the films from F5 (14 mg) to F6 $(28 \mathrm{mg})$ and further F7 (42 mg), consistently increased the acyclovir permeation. The amount of drug permeated across the membrane at 1 $\mathrm{h}$ was $20.65 \pm 4.26 \mu \mathrm{g} / \mathrm{cm}^{2}, 26.99 \pm 5.18 \mu \mathrm{g} / \mathrm{cm}^{2}, 44.70 \pm 8.63 \mu \mathrm{g} / \mathrm{cm}^{2}$ and $11.62 \pm 4.29 \mu \mathrm{g} / \mathrm{cm}^{2}$, in F5, F6, F7 and control, respectively. This observation also support the drug release studies in which the amount of drug release was higher when drug content was increased. Significant difference $(P<0.05)$ in acyclovir permeation between the formulations was observed from $4 \mathrm{~h}$ onwards. The flux values in F5, F6, F7 and control were $48.81 \pm 7.93 \mu \mathrm{g} / \mathrm{cm}^{2} / \mathrm{h}, 92.33 \pm 11.01 \mu \mathrm{g} / \mathrm{cm}^{2} / \mathrm{h}, 151.67 \pm 16.43 \mu \mathrm{g} /$ $\mathrm{cm}^{2} / \mathrm{h}$ and $22.60 \pm 4.23 \mu \mathrm{g} / \mathrm{cm}^{2} / \mathrm{h}$, respectively. Moreover, the enhancement in acyclovir permeation in F5, F6 and F7 loaded films were 2.16, 4.09 and 6.71 folds, when compared to control. Overall the data observed here substantiate our objective of enhancing the permeation of acyclovir by incorporating the drug in nanoparticles and further embedding into a buccal film.

\section{CONCLUSION}

The present study aimed to develop and evaluate buccoadhesive films to enhance acyclovir permeation. Acyclovir loaded biopolymeric nanoparticles were prepared and loaded into mucoadhesive films. The films were evaluated for physico-mechanical properties, in vitro and ex vivo studies. Drug loaded films (F5, F6 and F7) exhibited uniformity in drug content and desirable physico-mechanical properties such as $\mathrm{pH}$, film thickness, folding endurance, swelling capacity and mucoadhesive strength. Data observed with in vitro release studies substantiate the potential of de- veloped films to provide an adequate amount of acyclovir for diffusion across the buccal membrane. Permeation data further confirmed the potential of acyclovir loaded nanoparticles to transport into and across the buccal epithelium and provide adequate delivery of acyclovir for systemic therapy.

\section{CONFLICT OF INTEREST}

The authors report no conflict of interest.

\section{ACKNOWLEDGEMENT}

The authors thank the Deanship of Scientific Research, King Faisal University for funding this research project No. 175022.

\section{ABBREVIATIONS}

HEC: hydroxyethyl cellulose, PBS: phosphate buffered saline, PG: propylene glycol, PLGA: poly (D,L-lactide-co-glycolide)

\section{REFERENCES}

1. Gilhotra RM, Ikram M, Srivastava S, Gilhotra N. A clinical perspective on mucoadhesive buccal drug delivery systems. J Biomed Res. 2014;28:81-97.

2. Adhikari SN, Nayak BS, Nayak AK, Mohanty B. Formulation and evaluation of buccal patches for delivery of atenolol. AAPS Pharm Sci Tech. 2010;11:1038-44.

3. Sudhakar Y, Kuotsu K, Bandyopadhyay AK. Buccal bioadhesive drug delivery-a promising option for orally less efficient drugs. J Control Release. 2006;114:1540.

4. Kumria R, Gupta V, Bansal S, Wadhwa J, Nair AB. Oral buccoadhesive films of ondansetron, Development and evaluation. Int J Pharm Investig. 2013;3:112-8.

5. Jaipal A, Pandey MM, Charde SY, Sadhu N, Srinivas A, Prasad RG. Controlled release effervescent buccal discs of buspirone hydrochloride, in vitro and in vivo evaluation studies. Drug Deliv. 2016;23:452-8.

6. Lodhi M, Dubey A, Narayan R, Prabhu P, Priya S. Formulation and evaluation of buccal film of Ivabradine hydrochloride for the treatment of stable angina pectoris. Int J Pharm Investig. 2013;3(1):47-53

7. Giovino C, Ayensu I, Tetteh J, Boateng JS. Development and characterisation of chitosan films impregnated with insulin loaded PEG-b-PLA nanoparticles (NPs), a potential approach for buccal delivery of macromolecules. Int J Pharm. 2012;428:143-51.

8. Morales JO, Huang S, Williams RO $3^{\text {rd }}$, McConville JT. Films loaded with insulincoated nanoparticles (ICNP) as potential platforms for peptide buccal delivery. Colloids Surf B Biointerfaces. 2014;122:38-45

9. Al-Dhubiab BE, Nair AB, Kumria R, Attimarad M, Harsha S. Development and evaluation of buccal films impregnated with selegiline-loaded nanospheres. Drug Deliv. 2016;23:2154-62.

10. Cortesi R, Esposito E. Acyclovir delivery systems. Expert Opin Drug Deliv. 2008:5:1217-30.

11. Bhosale U, Kusum DV, Jain N. Formulation and optimization of mucoadhesive nanodrug delivery system of acyclovir. J Young Pharm. 2011;3(4):275-83.

12. Nair AB, Attimarad M, Al-Dhubiab BE, Wadhwa J, Harsha S, Ahmed M. Enhanced oral bioavailability of acyclovir by inclusion complex using hydroxypropyl$\beta$-cyclodextrin. Drug Deliv. 2014;21:540-7.

13. Wadhwa J, Nair A, Kumria R. Emulsion forming drug delivery system for lipophilic drugs. Acta Pol Pharm Drug Res. 2012;69(2):179-91.

14. Freitas S, Merkle HP, Gander B. Microencapsulation by solvent extraction/ evaporation, reviewing the state of the art of microsphere preparation process technology. J Control Release. 2005;102:313-32.

15. Versha $\mathrm{P}$, Ghosh B, Anroop B, Ramanjit M. Antimicrobial activity of Alstonia scholaris leaf extracts. Indian Drugs. 2003;40(7):412-3.

16. Al-Dhubiab BE, Nair AB, Kumria R, Attimarad M, Harsha S. Formulation and evaluation of nano based drug delivery system for the buccal delivery of acyclovir. Colloids Surf B Biointerfaces. 2015;136:878-84

17. Kumria R, Nair AB, Al-Dhubiab BE. Loratidine buccal films for allergic rhinitis, Development and evaluation. Drug Dev Ind Pharm. 2014;40:625-31.

18. Nair AB, Kumria R, Harsha S, Attimarad M, Al-Dhubiab BE, Alhaider IA. In vitro techniques to evaluate buccal films. J Control Release. 2013;166:10-21.

19. Nair AB, Gupta R, Kumria R, Jacob $S$, Attimarad M. Formulation and evaluation of enteric coated tablets of proton pump inhibitor. J Basic Clin Pharm 2010;1(4):215-21.

20. Nair AB, Sammeta SM, Vaka SRK, Murthy SN. A study on the effect of inorganic salts in transungual drug delivery of terbinafine. J Pharm Pharmacol. 2009;61:431-7.

21. Nair AB, Chakraborty B, Murthy SN. Effect of polyethylene glycols on the trans- 
ungual delivery of terbinafine. Curr Drug Deliv. 2010;7:407-14.

22. Arnal J, Gonzalez-Alvarez I, Bermejo M, Amidon GL, Junginger HE, Kopp S, et al. Biowaiver monographs for immediate release solid oral dosage forms, aciclovir. J Pharm Sci. 2008;97:5061-73.

23. Kumar A, Bali V, Kumar M, Pathak K. Comparative evaluation of porous venus nonporous mucoadhesive films as buccal delivery system of glibenclamide. AAPS Pharm SciTechnol. 2013;14:1321-32.

24. Kenechukwu FC, Momoh MA. Formulation, characterization and evaluation of the effect of polymer concentration on the release behavior of insulin-loaded Eudragit(@)-entrapped mucoadhesive microspheres. Int J Pharm Investig. 2016;6(2):69-77.
25. Prasanna RI, Sankari KU. Design, evaluation and in vitro-in vivo correlation of glibenclamide buccoadhesive films. Int J Pharm Investig. 2012;2(1):26-33.

26. Kumria R, Nair AB, Goomber G, Gupta S. Buccal films of prednisolone with enhanced bioavailability. Drug Deliv. 2016;23:471-8.

27. Mudshinge SR, Deore AB, Patil S, Bhalgat CM. Nanoparticles, Emerging carriers for drug delivery. Saudi Pharm J. 2011;19:129-41.

28. Nair AB, Sammeta SM, Kim HD, Chakraborty B, Friden PM, Murthy SN. Alteration of the diffusional barrier property of the nail leads to greater terbinafine drug loading and permeation. Int J Pharm. 2009;375:22-7.

29. Nair AB, Jacob S. A simple practice guide for dose conversion between animals and human. J Basic Clin Pharm. 2016;7:27-31.

Article History: Submission Date: 13-08-16; Revision Date: 30-09-16; Accepted Date: 01-10-16.

Cite this article: Nair AB, Al-ghannam AA, Al-Dhubiab BE, Hasan AA. Mucoadhesive Film Embedded with Acyclovir Loaded Biopolymeric Nanoparticles: In vitro Studies. J Young Pharm. 2017;9(1):100-5. 